\title{
How I Treat
}

\section{How I Treat Renal Cell Carcinoma}

\section{Ronald M. Bukowski, MD}

Renal cell carcinoma (RCC) accounts for 3\% of malignant tumors and is the sixth leading cause of cancer death in the United States. An estimated 51,000 new renal tumors were diagnosed in 2006, with 13,000 deaths. ${ }^{1}$ It is most common in the sixth decade of life, and a male to female predominance of 1.6 to 1.0 is present. Current therapeutic approaches for treatment of patients with metastatic RCC utilize knowledge of histology, molecular abnormalities, clinical prognostic factors, and the effects of available agents.

\section{Histologic Subsets}

Epithelial RCC includes various histologic subtypes, each having unique morphologic and genetic characteristics. Clearcell RCC is the most common, accounts for $70 \%$, and arises from the proximal convoluted tubule. Importantly, $60 \%$ to $80 \%$ of sporadic clear-cell RCCs are associated with defects in the $V H L$ gene. ${ }^{2}$ In contrast, RCC in patients with von Hippel-Lindau (VHL) syndrome all have VHL gene mutations. The remaining subtypes of epithelial RCC are collectively referred to as nonclear cell carcinomas, and do not have abnormalities of this gene. In this group, papillary RCC is the most common type (10\% to $15 \%)$. Understanding histologic subtypes and associated molecular alterations has provided the framework for the development of diseasespecific therapy.

\section{Molecular Biology Clear Cell Carcinoma}

In sporadic clear-cell RCC, both the maternal and paternal $V H L$ alleles are inactivated by acquired mutations. The VHL protein functions as a tumor suppressor, and is responsible for ubiquination and proteasome degradation ${ }^{3}$ of hypoxiainducible factor (HIF)- $\alpha$ regulator of the hypoxic response. Under hypoxia, or when VHL protein is nonfunctional, it does not bind and inactivate HIF- $\alpha$, resulting in its accumulation. This in turn activates transcription of a variety of hypoxia-inducible genes, including vascular endothelial growth factor (VEGF), platelet-derived growth factor- $\beta$ (PDGF $\beta)$, transforming growth factor $-\alpha$, and erythryopoietin.

Clear-cell cancers are highly vascular, in part secondary to stimulation of tumor associated angiogenesis. VHL protein plays a pivotal role in neoangiogenesis, but loss of $V H L$ gene function results in enhanced secretion of VEGF, PDGF, and the resulting vascular phenotype.

\section{Prognostic Classification: Advanced RCC}

Retrospective analysis of patients with untreated metastatic RCC has identified clinical characteristics associated with differences in prognosis. An initial model was developed at Memorial Sloan-Kettering Cancer Center (New York, New York), and validated at the Cleveland Clinic (Cleveland, Ohio). 4,5 These risk criteria have now been utilized in a series of phase III clinical trials. The five factors include low Karnofsky performance status ( $<80 \%$ ), low serum hemoglobin, high corrected calcium, elevated lactate dehydrogenase, and short disease-free interval ( $<1$ year). Prognostic groups were defined as favorable (no factors), intermediate ( $\leq 2$ factors), and poor ( $\geq 3$ factors), with median overall survival (OS) of 29.6, 13.8, and 4.9 months, respectively. ${ }^{4}$

\section{Therapy for Advanced RCC: Historical Perspective}

In the past, treatment with interleukin (IL)-2 and/or recombinant interferon alfa (IFN $\alpha$ ) have been the standard approaches for advanced RCC.

Overall response rates (ORR) were improved with high-dose IL-2 (21\%) compared with low-dose intravenous IL-2 (11\%), or subcutaneous IL-2 (10\%), however, no progression-free survival (PFS) or OS advantages were observed. ${ }^{6}$ A second study utilizing high-dose IL-2 reported an ORR of $23.2 \%$, and $9.9 \%$ with subcutaneous IL-2 plus IFN $\alpha$, without a PFS or OS advantage. ${ }^{7}$ IFN $\alpha$ monotherapy has been the standard of care for advanced RCC, in view of the modest survival benefit identified in recent trials ${ }^{8}$ and meta-analysis. ${ }^{9}$ In view of the toxicity and limited benefit of the IL- 2 and IFN $\alpha$ cytokines, new treatments were needed.

\section{Development of a New Treatment Paradigm}

The molecular abnormalities in clear-cell RCC suggested angiogenesis inhibitors should be investigated. Bevacizumab is a monoclonal antibody binding all VEGF isoforms, ${ }^{10}$ which inhibits VEGF signaling. A phase III placebo controlled trial (AVOREN) recently investigated IFN $\alpha 9$ million units three times per week with or without bevacizumab $(10 \mathrm{mg} / \mathrm{kg}$ every 2 weeks). The combination improved the ORR (31\%v13\%; $P=.0001)$ and PFS (10.2 v 5.4 months; hazard ratio [HR] $=0.63 ; P=.0001) .{ }^{11}$

The oral agents sunitinib and sorafenib that inhibit multiple tyrosine kinases (VEGFR-2, PDGFR, c-kit, Flt3) have also been studied. A randomized trial ${ }^{12}$ comparing sunitinib to 
Figure 1. The clinical approach to patients with advanced/metastatic renal cell carcinoma is illustrated. The various treatment choices are initially guided by the histologic subtype, prior therapy if any, and the whether the patient presents with metachrous or synchronous metastases. Subsequently, the clinical prognostic criteria and comorbid medical status provides direction. The therapeutic choices include those discussed in the text; in addition, potential roles of radiation therapy for symptomatic and CNS metastatases, as well as the use of a bisphosphonate, such as zoledronic acid, in the setting of osseous metastases is included. RCC, renal cell carcinoma; Mets, metastasis; VEGF, vascular endothelial growth factor; IFN $\alpha$, interferon alfa; HD, high dose; IL, interleukin.

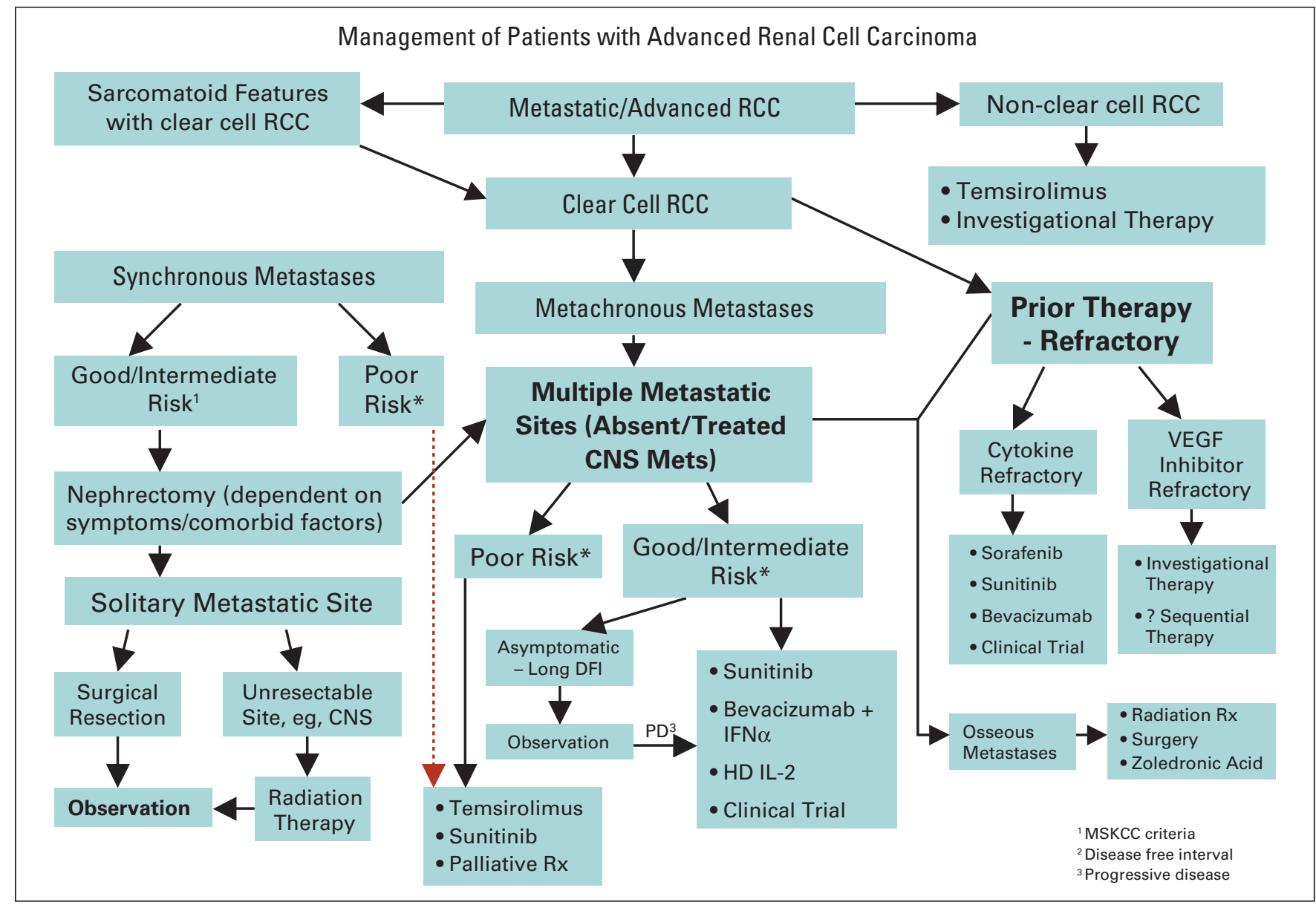

IFN $\alpha$ in 750 treatment naïve patients has been reported. The median PFS was 11 months in the sunitinib arm compared with 5 months in the IFN $\alpha$ arm (HR, $0.42 ; P<.001$ ). The ORR was $31 \%$ for patients receiving sunitinib compared with $6 \%$ in the IFN arm $(P<.001)$. This trial established sunitinib as a reference standard for treatment of advanced RCC.

In contrast, sorafenib ${ }^{13}$ was investigated in 905 cytokine refractory patients with clear-cell RCC utilizing a randomized and blinded phase III design. Patients received either sorafenib (400 mg twice per day) or placebo. A preplanned interim analysis demonstrated significant $(P=.000001)$ prolongation of PFS in patients treated with sorafenib $(5.8 v$ 2.8 months). The ORR for sorafenib was $10 \%$ compared with $2 \%$ for the placebo, however, more than $70 \%$ of sorafenib patients had some decrease in tumor size. The study was terminated after the analysis, and all patients were crossed over to sorafenib. The final survival analysis suggests an effect of the crossover.
The mammalian target of rapamycin (mTOR), a polypeptide kinase, is also therapeutic target in RCC. $\mathrm{mTOR}$ is an upstream activator of HIF, and stabilizes, prevents degradation, and thereby increases HIF activity. ${ }^{14}$ Temsirolimus, an inhibitor of this mTOR, has been studied in a phase III randomized trial. ${ }^{15}$ Temsirolimus $(25 \mathrm{mg} / \mathrm{kg} /$ week intravenously), IFN $\alpha$ monotherapy, or the combination were investigated as first-line treatment in 626 poor prognostic patients. ${ }^{16}$ The definition of poor-risk required three or more risk factors, including metastases to multiple organs.

Temsirolimus monotherapy improved median OS (10.9 $v 7.3$ months; $\mathrm{HR}=0.63 ; P=.0068)$ and PFS compared with IFN $\alpha$. An updated subset analysis ${ }^{16}$ reported the effects of histology and prognostic factors. Median OS and PFS were increased in temsirolimus patients regardless of histologic subtype, and this was most pronounced in the nonclear subset. For patients in the intermediate Memorial SloanKettering Cancer Center prognostic group ${ }^{4}(\leq 2$ risk factors) 
no effect was noted, with only the poor-risk temsirolimus patients showing improved median OS.

\section{Management of Advanced RCC}

The results of these clinical trials clearly demonstrate agents inhibiting the VEGF pathway have significant antitumor effects in patients with advanced clear-cell RCC. These can now be utilized to plan therapy for patients with advanced RCC. In Figure 1, a flow diagram was constructed to illustrate an overall approach. Therapeutic choices are guided by the clinical presentation and prognostic factors, histologic variety, and prior therapy status. The underlying basis of the alogrithm utilizes the new treatment paradigm that has been

\section{References}

1. American Cancer Society: Cancer Facts \& Figures 2006. Atlanta, GA, American Cancer Society, 2006

2. Linehan WM, Vasselli J, Srinivasan R, et al: Genetic basis of cancer of the kidney: Disease-specific approaches to therapy. Clin Cancer Res 10:6282s6289s, 2004

3. Kim WY, Kaelin WG: The role of VHL gene mutation in human cancer. J Clin Oncol 22:4991-5004, 2004

4. Motzer RJ, Bacik J, Murphy BA, et al: Interferon-alfa as a comparative treatment for clinical trials of new therapies against advanced renal cell carcinoma. J Clin Oncol 20:289-296, 2002

5. Mekhail TM, Abou-Jawde RM, BouMerhi G, et al: Validation and extension of the Memorial Sloan-Kettering prognostic factors model for survival in patients with previously untreated metastatic renal cell carcinoma. J Clin Oncol 23:832-841, 2005

6. Yang JC, Sherry RM, Steinberg SM, et al: Randomized study of high-dose and low-dose interleukin-2 in patients with metastatic renal cancer. J Clin Oncol 21: 3127-3132, 2003

7. McDermott DF, Regan MM, Clark JI, et al: Randomized phase III trial of highdose interleukin-2 versus subcutaneous interleukin-2 and interferon in patients with metastatic renal cell carcinoma. J Clin Oncol 23:133-141, 2005 developed for RCC over the past 5 years. Clinical trials continue to play an important part of this approach, and will be instrumental in further refining treatment, identifying the role of combinations, sequential VEGF inhibitors, and importantly identifying the utility of novel agents such as RAD-001, axitinib, and pazopanib.

Ronald Bukowski, MD, is Director of Experimental Therapeutics, Cleveland Clinic Taussig Cancer Center and Professor of Medicine at Cleveland Clinic Lerner College of Medicine of Case Western Reserve University, Cleveland, Ohio; bukowsr@ccf.org.

\section{DOI: 10.1200/JOP.0838501}

8. Collaborators MRC: Interferon alfa and survival in metastatic renal carcinoma: Early results of a randomized controlled trial. Lancet 353:14-17, 1999

9. Coppin C, Porzsolt F, Awa A, et al: Immunotherapy for advanced renal cell cancer. Cochrane Database Syst Rev 1:CD001425, 2005

10. Ferrara N, Gerber HP, LeCouter J: The biology of VEGF and its receptors. Nat Med 6:669-676, 2003

11. Escudier B, Pluzanka A, Koralewski $P$, et al: Bevacizumab plus interferon-2a for treatment of metastatic renal cell carcinoma: A randomized, double-blind phase III trial. Lancet 370:2303-2311, 2007

12. Motzer RJ, Hutson TE, Tomczak $P$, et al: Sunitinib versus interferon alfa in metastatic renal-cell carcinoma. N Engl J Med 356:115-124, 2007

13. Escudier B, Eisen T, Stadler WM, et al: Sorafenib in advanced clear-cell renal-cell carcinoma. N Engl J Med 356:125-134, 2007

14. Dutcher JP: Mammalian target of rapamycin inhibition. Clin Cancer Res 10: 6382S-6387S, 2004

15. Hudes G, Carducci M, Tomczak P, et al: Temsirolimus, interferon alfa, or both for advanced renal-cell carcinoma. N Engl J Med 356:2271-2281, 2007

16. Dutcher JP, Szczylik C, Tannir N, et al: Correlation of survival with tumor histology, age, and prognostic factor risk group for previously untreated patients with advanced renal cell carcinoma (adv RCC) receiving temsirolimus (TEMSR) or interferon-alpha (IFN). J Clin Oncol 25:243s, 2007 (abstr 5033)

\section{REGISTER FOR THE 2008 BREAST CANCER SYMPOSIUM}

The 2008 Breast Cancer Symposium (September 5-7, 2008, Washington, DC) has expanded this year to a $21 / 2$ day event to provide more feature presentations on the latest multidisciplinary research from selected, theme-based translational and clinical abstracts, as well as related educational sessions. This symposium offers an opportunity for clinically relevant, in-depth discussions of how and when to translate new findings into patient care and how to be more selective about breast cancer therapy. Register by August 1, 2008 for discounted rates. To register, visit www.breastcasymposium.org.

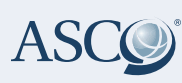

American Society of Clinical Oncology 